\title{
In Reply: Successful Extubation After Weaning Failure by Non-invasive Ventilation in Patients With Neurmuscular Disease - Do We Appreciate the Bigger Picture?
}

\author{
Sun Mi Kim, MD ${ }^{1}$, Yu Hui Won, $\mathrm{MD}, \mathrm{PhD}^{2}$, Seong-Woong Kang, $\mathrm{MD}$, $\mathrm{PhD}^{1,3}$
}

\begin{abstract}
${ }^{1}$ Department of Medicine, Graduate School of Yonsei University, Seoul; ${ }^{2}$ Department of Physical Medicine and Rehabilitation, Research Institute of Clinical Medicine of Chonbuk National University-Biomedical Research Institute of Chonbuk National University Hospital, Jeonju; ${ }^{3}$ Department of Rehabilitation Medicine, Pulmonary Rehabilitation Center, Gangnam Severance Hospital, Rehabilitation Institute of Neuromuscular Disease, Yonsei University College of Medicine, Seoul, Korea
\end{abstract}

We appreciate the writer's interest and comment on the recently published article "Successful Extubation After Weaning Failure by Noninvasive Ventilation in Patients With Neuromuscular Disease: Case Series." We have already mentioned in the original article that there is growing evidence that noninvasive ventilation (NIV) helps wean patients (afflicted with chronic respiratory disease) off ventilation (mainly those with chronic obstructive pulmonary disease) [1-3]. Also in our recent publication, by means of employing mechanically-assisted coughing and NIV, 60 subjects with high cervical spinal cord injuries with tracheostomy succeeded in decannulation [4]. However, the efficacy of NIV in patients with neuromuscular disease (NMD) suffering from weaning failure has not yet been assessed in a large case study.

Acute illness increase respiratory workload, in turn causing decompensation of the respiratory musculature in patients afflicted with NMD. Eventually, these pathologies produce need for endotracheal intubation and invasive mechanical ventilation. Performing extubation in these subjects can be more challenging due to the advanced degradation of the respiratory musculature. The NIV support is especially important for those patients who develop respiratory muscle fatigue due to the increased muscle work load after extubation, in order to obviate the necessity of re-intubation [5].

As the writer has commented, there had been major changes and large improvements in pulmonary rehabilitation practice in patients who are under critical care. Procedures have become more sophisticated and systematic and with the application of new facial interfaces, new monitoring systems of transcutaneous partial pressure of carbon dioxide and the renewed development of various portable mechanical ventilators have evolved. However, the aforementioned developments have not altered, or appreciably changed, the main flow of the practice which is to say, successful extubation is possible with ventilation support using mechanical ventilation in a noninva-

Corresponding author: Seong-Woong Kang

Department of Rehabilitation Medicine, Pulmonary Rehabilitation Center, Gangnam Severance Hospital, Rehabilitation Institute of Neuromuscular Disease, Yonsei University College of Medicine, 211 Eonju-ro, Gangnam-gu, Seoul 06273, Korea. Tel: +82-2-2019-3492, Fax: +82-2-3463-7585, E-mail: kswoong@yuhs.ac

ORCID: Sun Mi Kim (http://orcid.org/0000-0003-4562-2869); Yu Hui Won (http://orcid.org/0000-0003-2007-9652); Seong-Woong Kang (http://orcid. org/0000-0002-7279-3893).

(c) This is an open-access article distributed under the terms of the Creative Commons Attribution Non-Commercial License (http://creativecommons.org/ licenses/by-nc/4.0) which permits unrestricted noncommercial use, distribution, and reproduction in any medium, provided the original work is properly cited. Copyright (C) 2017 by Korean Academy of Rehabilitation Medicine 
sive way, and secretion control using mechanical cough assist $[5,6]$.

As mentioned in writer's comment, not providing the information on other important demographic datasuch as the actual number of patients with NMD successfully extubated at the first attempt, with or without the need for NIV (we do not get informed of these patients at all since they are managed successfully at the first attempt in other departments) - was one of the limitations of this study. As we exclusively collected data relating to NIV supported-successful extubation cases after extubation failure and re-intubation only, the sample size was eventually winnowed down to a relatively small size, and we chose to report these as series of cases instead of an original article. In retrospect, it also seems unfortunate that we neglected to collect the other demographic data that the writers commented upon.

Not concurrently, however, the authors conducted a study on the effect of pulmonary rehabilitation of NMD patients with very low vital capacity who had undergone scoliosis correction operation [7]. Among the total of 24 patients, only three patients were not using the mechanical ventilator prior to the scoliosis operation. Although some patients experienced delayed extubation, every patient was successfully extubated on the first attempt. Among the three patients who were not using the ventilator, one patient was newly applied to NIV during extubation, and the other two patients were extubated without the support of NIV.

It may be premature to suggest any hard indication for the technique through our original report, since we were unable to collect the total number of patients who were extubated with or without NIV support during that same period of time, and thus making it hard to calculate a precise failure rate of this technique. Even so, we believe that showing variable cases of patients who were managed with NIV support still has clinical value since tracheostomy is frequently chosen to be performed in patients with advanced respiratory dysfunction (as was the case in our original case report).

There was lack of consensus and no formal concept or protocol on using NIV as a bridging technique during extubation procedure in those with respiratory failure, especially in the early period of this study. Thus, performing tracheostomy right after extubation failure was more common in patients who were treated in other depart- ments. Moreover, those patients were discharged without references for consultations to appropriate department of rehabilitation, making it even more difficult for us to follow the exact count. Recently, however, attempts on trying NIV prior to direct tracheostomy has grown in number compared, to the early 2000s. Along with the increased experience of pulmonary rehabilitation of this pulmonary rehabilitation center, we anticipate that the success rate of the extubation with NIV support will also likely increase. We believe that our next goal should focus on advancing the genuine usefulness of this technique, with the support of exact success and failure rate data.

We followed up in continuing to assess the longer term data pertinent to each of the patients who were included in the original study through their medical records. Of the total of eighteen patients included in this study, only one patient with amyotrophic lateral sclerosis (ALS) was lost to follow-up before 1 year had elapsed since the extubation. He maintained NIV until 10 months after extubation. However, the patient failed to visit the hospital after that time and was eventually lost to follow-up. Another patient, concurrently afflicted with ALS, failed to survive until the 1 year mark post-extubation, having succumbed to other medical problems after 5 months. The remaining sixteen patients survived until at least 1 year post-extubation, without re-intubation or tracheostomy. Three patients died before 2013; two were afflicted with Duchenne muscular dystrophy and died, respectively, 64 months and 74 months, after extubation, and the other one was afflicted with spinal musculature atrophy and died 24 months after extubation. If one excludes the one patient with ALS who was lost to follow-up, sixteen of seventeen survived, recording $94.1 \%$ of a 1-year survival among these patients and all the seventeen patients succeeded in maintaining NIV.

\section{REFERENCES}

1. Ferrer M, Sellares J, Valencia M, Carrillo A, Gonzalez G, Badia JR, et al. Non-invasive ventilation after extubation in hypercapnic patients with chronic respiratory disorders: randomised controlled trial. Lancet 2009;374:1082-8.

2. Esteban A, Frutos-Vivar F, Ferguson ND, Arabi Y, Apezteguia C, Gonzalez M, et al. Noninvasive positivepressure ventilation for respiratory failure after extu- 
bation. N Engl J Med 2004;350:2452-60.

3. Nava S, Gregoretti C, Fanfulla F, Squadrone E, Grassi M, Carlucci A, et al. Noninvasive ventilation to prevent respiratory failure after extubation in high-risk patients. Crit Care Med 2005;33:2465-70.

4. Kim DH, Kang SW, Choi WA, Oh HJ. Successful tracheostomy decannulation after complete or sensory incomplete cervical spinal cord injury. Spinal Cord 2017;55:601-5.

5. Bach JR, Goncalves MR, Hon A, Ishikawa Y, De Vito EL, Prado F, et al. Changing trends in the manage- ment of end-stage neuromuscular respiratory muscle failure: recommendations of an international consensus. Am J Phys Med Rehabil 2013;92:267-77.

6. Bach JR, Goncalves MR, Hamdani I, Winck JC. Extubation of patients with neuromuscular weakness: a new management paradigm. Chest 2010;137:1033-9.

7. Lee JW, Won YH, Kim DH, Choi WA, Bach JR, Kim DJ, et al. Pulmonary rehabilitation to decrease perioperative risks of spinal fusion for patients with neuromuscular scoliosis and low vital capacity. Eur J Phys Rehabil Med 2016;52:28-35. 